\title{
Mechanical design and analysis of an eight-pole superconducting vector magnet for soft x-ray magnetic dichroism measurements
}

\author{
D. Arbelaez, A. Black, S.O. Prestemon, S. Wang, J. Chen, and E. Arenholz, Senior Member, IEEE
}

\begin{abstract}
An eight-pole superconducting magnet is being developed for soft $x$-ray magnetic dichroism (XMD) experiments at the Advanced Light Source, Lawrence Berkley National Laboratory (LBNL). Eight conical $\mathrm{Nb}_{3} \mathrm{Sn}$ coils with Holmium poles are arranged in octahedral symmetry to form four dipole pairs that provide magnetic fields of up to $5 \mathrm{~T}$ in any direction relative to the incoming $x$-ray beam. The dimensions of the magnet yoke as well as pole taper, diameter, and length were optimized for maximum peak field in the magnet center using the software package TOSCA. The structural analysis of the magnet is performed using ANSYS with the coil properties derived using a numerical homogenization scheme. It is found that the use of orthotropic material properties for the coil has an important influence in the design of the magnet.
\end{abstract}

Index Terms-soft $\mathrm{x}$-rays, magnetic dichroism, superconducting magnet.

\section{INTRODUCTION}

$\mathbf{I}$ $\mathrm{N}$ order to take full advantage of soft $\mathrm{x}$-ray magnetic dichroism (XMD) measurements, magnetic fields that can be oriented in any direction relative to the incoming $\mathrm{X}$-ray beam in an $\mathrm{x}$-ray absorption experiment are required. A resistive eight pole magnet that is currently installed at beamline 4.0.2 of the Advanced Light Source (ALS), Lawrence Berkley National Laboratory (LBNL), has been used to demonstrate that the combination of $\mathrm{x}$-ray magnetic circular and linear dichroism measurements as well as the XMD angular dependence provide information not accessible by other means [1]. While this device has been successfully used to study many materials, its peak magnetic field of $0.9 \mathrm{~T}$ is limiting the systems that can be investigated. In order to align the magnetic moments along the hard magnetization direction in many oxide hetero- and nanostructures, magnetic fields near $5 \mathrm{~T}$ are required. An eight-pole superconducting magnet is being developed at LBNL and Wang NMR Inc. to overcome the limitations of the resitive magnet device. The planned superconducting magnet is composed of eight conical $\mathrm{Nb}_{3} \mathrm{Sn}$ coils with Holmium poles that are arranged in an octahedral pattern to form four dipole pairs. Figure 1 shows a CAD model of the magnets central set up including the internal support structure, the $\mathrm{Nb}_{3} \mathrm{Sn}$ coils, the poles, and the magnet yokes. The internal monolithic structure contains eight pockets to support the $\mathrm{Nb}_{3} \mathrm{Sn}$ coils. A Holmium pole is inserted into each

Manuscript received 3 August 2010. This work was supported by ...

D. Arbelaez, A. Black, S. Prestemon and E. Arenholz, are with Lawrence Berkeley National Laboratory, Berkeley, CA, 94720 USA (e-mail: darbelaez@lbl.gov).

S. Wang and J. Chen are with Wang NMR Inc. coil. The magnet yoke consists of eight parts that are attached to the Ho poles. These pieces are bolted onto the monolithic central magnet structure. Figure 2 shows a cross-section of the magnet which contains a six-way cross that allows access for the sample, the $\mathrm{x}$-ray beam, as well as detectors.

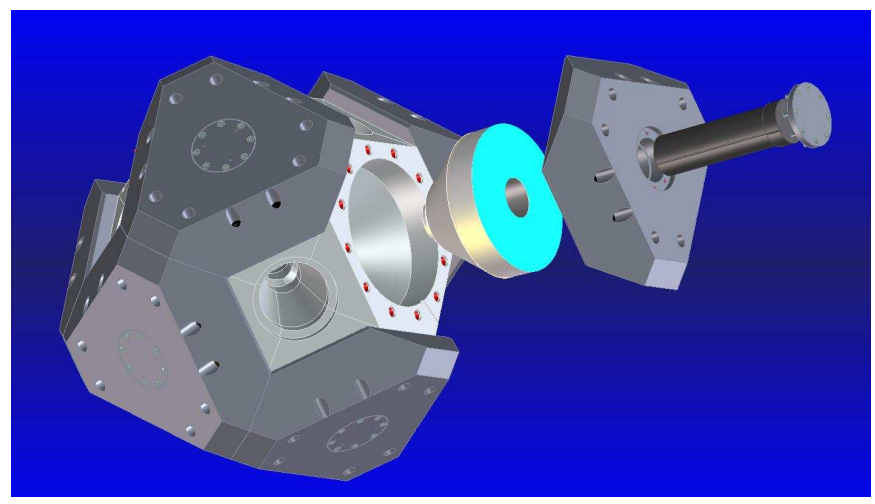

Fig. 1. CAD model of the magnet's central structure including the internal monolithic support piece, the $\mathrm{Nb}_{3} \mathrm{Sn}$ coils, the Holmium poles, and the yoke pieces.

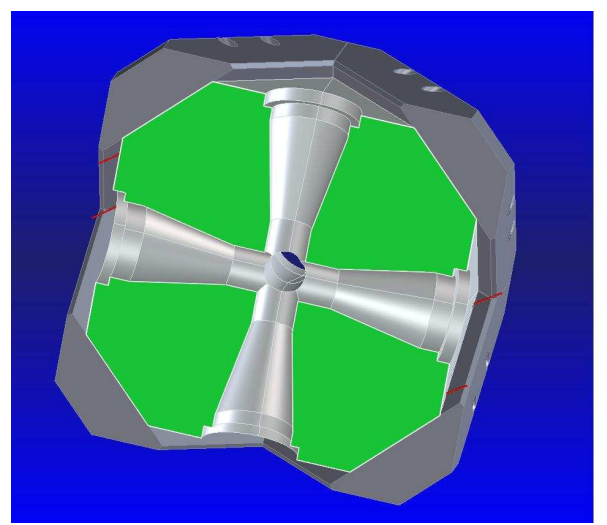

Fig. 2. Cross-section of the magnet showing the six-way cross that allows access to the sample from different directions for magnetic dichroism measurements.

In this paper the structural analysis of the magnet is discussed. The mechanical analysis of the magnet is performed with ANSYS with applied magnetic forces that are computed using TOSCA. The effective material properties for the wire wound coils are computed with a computational homogenization scheme. In [2], a similar scheme was used for coils 
composed of Rutherford cables. Using the computed properties, analytical and computational models for the deformation and stress state in the coil are presented. The effect of the orthotropic properties of the coil on the contact state between the coil and the support structure and poles is also discussed.

\section{EFFECTIVE COIL PROPERTIES}

In this section, a computational homogenization scheme that is used to determine the effective properties of the "coil material" is presented. Figure 3 shows an example of a calculation over the periodic unit cell that is used to determine the effective properties. The unit cell includes the strand (inner circle), the strand insulation (outer ring surrounding the strand), and the interstitial epoxy. The "coil material" is

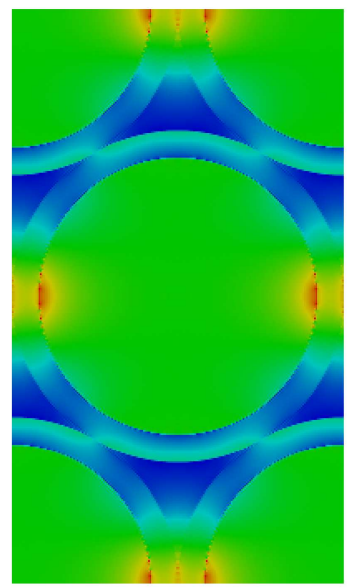

Fig. 3. Example of a unit cell calculation for the coil material. The unit cell includes the strand (inner circle), the strand insulation (outer ring), and the interstitial epoxy. The colors indicate different strain states.

created by superimposing unit cells in an infinite space, which is expected to be a good approximation for a coil with a large number of windings. For this model, periodic boundary conditions are appropriate. The periodic boundary condition is described by

$$
\mathbf{u}^{+}-\mathbf{u}^{-}=\mathcal{E}\left(\mathbf{X}^{+}-\mathbf{X}^{-}\right),
$$

where $\mathbf{u}^{+}$and $\mathbf{u}^{-}$represent the displacements of two points directly opposite to one another on the periodic surfaces, $\mathbf{X}^{+}$ and $\mathbf{X}^{-}$are their corresponding positions, and $\mathcal{E}$ is the average strain imposed over the unit cell. In order to compute the effective properties, six different appropriate loading conditions can be applied to determine the effective elasticity tensor. Writing the stress strain linear elastic relation in engineering notation:

$$
\left\{\begin{array}{c}
\sigma_{x} \\
\sigma_{y} \\
\sigma_{z} \\
\tau_{x y} \\
\tau_{y z} \\
\tau_{z x}
\end{array}\right\}=\left[\begin{array}{llllll}
C_{11} & C_{12} & \cdot & \cdot & C_{16} \\
C_{12} & C_{22} & & & C_{26} \\
C_{13} & C_{23} & & & C_{36} \\
C_{14} & C_{24} & & & C_{46} \\
C_{15} & C_{25} & & & C_{56} \\
C_{16} & C_{26} & . & . & \cdot & C_{66}
\end{array}\right]\left\{\begin{array}{c}
\varepsilon_{x} \\
\varepsilon_{y} \\
\varepsilon_{z} \\
\gamma_{x y} \\
\gamma_{y z} \\
\gamma_{z x}
\end{array}\right\}
$$

This equations shows that if only one non-zero value of the strain components is chosen, then the corresponding column of the elasticity matrix can be determined by computing the stress tensor for that applied strain state. To extract the effective properties of the unit cell, six different average strain states (imposed by $\mathcal{E}$ in equation 1) over the defined domain are applied, and the corresponding average stress states are calculated. For example, to compute the first column of the average elasticity matrix, a value of $\left\langle\varepsilon_{x}\right\rangle_{\Omega}$ is imposed with the other strain components set to zero and the average stress $\langle\boldsymbol{\sigma}\rangle_{\Omega}$ is computed. The operator $\langle\cdot\rangle_{\Omega}$ denotes the average over the domain $\Omega$ which corresponds to the unit cell.

Table I shows the material properties that are used to calculate the effective coil properties, where the abbreviations Str., Ep., and Ins. stand for strand, epoxy, and insulation respectively. For the insulation, the subscripts for the Young's moduli, $E$, the Poisson's ratios, $\nu$, the shear moduli, $G$, and the total thermal strain from $295 \mathrm{~K}$ to $4.2 \mathrm{~K}, \varepsilon^{t h}$, correspond to the three principal directions. For the epoxy and the strand, the materials are assumed to be isotropic; therefore, only two elastic constants are needed. The Poisson's ratios, $\nu_{i j}$ are defined as the negative of the transverse strain in the $j$-direction over the strain in the $i$-direction, when stress is applied only in the $i$-direction. The remaining Poisson's ratios can be found using the relation

$$
\nu_{j i}=\nu_{i j} \frac{E_{j}}{E_{i}} .
$$

The strand properties are determined using a rule of mixtures approach with the individual material properties for $\mathrm{Cu}$ and $\mathrm{Nb}_{3} \mathrm{Sn}$ obtained from the work by Mitchell [3]. The epoxy properties are obtained from the CTD-101 data sheet [4] where the shear modulus is given at $77 \mathrm{~K}$ and $295 \mathrm{~K}$. Assuming a Poisson's ratio of 0.3 , the Young's modulus is determined. Note that the $77 \mathrm{~K}$ shear modulus is used at $4.2 \mathrm{~K}$ since no measured value is given for this temperature. The value for the thermal expansion of epoxy was obtained from the program CRYOCOMP. For the insulation layer, properties for glass fiber and epoxy composites, which are obtained from two different sources, are used. For the shear moduli and the Young's modulus in the 3 direction, data was taken from the CTD-101 data sheet for a combination of 50\% and 60\% s-glass reinforced epoxies. Unfortunately, data is not provided for the Young's moduli in the fiber directions. Therefore, for the 1 and 2 directions, data for G10 is used. The Young's moduli are taken to be equal to the average of the moduli in the fill and warp directions, where this data was obtained from the USPAS 2001 notes on superconducting accelerator magnets by Goodzeit [5]. The data for thermal expansion of the insulation layer is taken from CRYOCOMP using G10 as the material. Clearly the accuracy of the individual material properties will have a large impact on the calculated homogenized properties.

Using the properties in Table I, the effective properties of the coil can be determined. Since the insulation layer is anisotropic, the principal directions have to be specified within an element. In this case the 3 direction corresponds to the radial direction from the center of the strand on which the insulation lies. Since the properties are taken as transversely isotropic, defining only one direction is sufficient. Table II shows the calculated effective properties for the coil. These 
TABLE I

ASSUMED PROPERTIES FOR THE CONSTITUENT MATERIALS IN THE COIL

\begin{tabular}{lllllll}
\hline \hline & \multicolumn{3}{c}{$295 \mathrm{~K}$} & \multicolumn{3}{c}{$4.2 \mathrm{~K}$} \\
\cline { 2 - 7 } & Str. & Ep. & Ins. & Str. & Ep. & Ins. \\
\hline$E_{1}(\mathrm{GPa})$ & 130 & 3.6 & 25 & 130 & 6.2 & 32 \\
$E_{2}(\mathrm{GPa})$ & - & - & 25 & - & - & 32 \\
$E_{3}(\mathrm{GPa})$ & - & - & 13 & - & - & 16 \\
$\nu_{12}$ & 0.34 & 0.30 & 0.30 & 0.34 & 0.30 & 0.30 \\
$\nu_{23}$ & - & - & 0.30 & - & - & 0.30 \\
$\nu_{31}$ & - & - & 0.16 & - & - & 0.16 \\
$G_{12}(\mathrm{GPa})$ & - & - & 23 & - & - & 29 \\
$G_{23}(\mathrm{GPa})$ & - & - & 5.2 & - & - & 6.6 \\
$G_{31}(\mathrm{GPa})$ & - & - & 5.2 & - & - & 6.6 \\
$\varepsilon_{1}^{t h}(\%)$ & 0.25 & 1.4 & 0.27 & & & \\
$\varepsilon_{2}^{t h}(\%)$ & - & - & 0.27 & & & \\
$\varepsilon_{3}^{t h}(\%)$ & - & - & 0.76 & & & \\
\hline \hline
\end{tabular}

TABLE II

CALCULATED PROPERTIES FOR THE COIL MATERIAL

\begin{tabular}{lll}
\hline \hline & $295 \mathrm{~K}$ & $4.2 \mathrm{~K}$ \\
\hline$E_{L}(\mathrm{GPa})$ & 88 & 90 \\
$E_{T}(\mathrm{GPa})$ & 31 & 40 \\
$\nu_{12}$ & 0.28 & 0.29 \\
$\nu_{31}$ & 0.33 & 0.33 \\
$G_{31}(\mathrm{GPa})$ & 13 & 17 \\
$\varepsilon_{L}^{t h}(\%)$ & 0.26 & \\
$\varepsilon_{T}^{t h}(\%)$ & 0.41 & \\
\hline \hline
\end{tabular}

properties are transversely isotropic; therefore, only longitudinal ( $\mathrm{L}$ or 3 ) and transverse ( $\mathrm{T}$ or 1-2 plane) components are necessary to fully describe the material behavior.

\section{FreE Thermal CONTRACTION OF THE ORTHOTROPIC COIL}

$\mathrm{The} \mathrm{Nb}_{3} \mathrm{Sn}$ coil has orthotropic properties in a cylindrical coordinate system defined by the three directions $r, \theta$, and $z$. After cool down, due to the different thermal expansion coefficients in the $r$ and $\theta$ directions, the coil is no longer in a stress free state even if there are no loads applied on the boundaries. This is due to the geometry of the coil which only allows radial displacements due to its axisymmetric nature. The solution for the displacement and stress in an orthotropic coil with negligible axial stress can be determined analytically by using the strain displacement relations, the stress strain relation, and enforcing that the stress tensor is divergence free. The radial displacement in the orthotropic coil, as a function of radial position, is determined to be

$$
u(r)=D_{1} r^{k}-D_{2} r^{-k}+\frac{\left(1-\nu_{\theta r}\right) \varepsilon_{r}^{t h}+\left(\nu_{\theta r}-k^{2}\right) \varepsilon_{\theta}^{t h}}{1-k^{2}} r
$$

where $D_{1}$ and $D_{2}$ are constants determined from the boundary conditions and $k^{2}=E_{\theta} / E_{r}$. For the coil geometry, the properties in the $\theta$ and $z$ directions in equation 4 correspond to the properties in the 3 and 1 directions in table II respectively. The stress state in the coil is given by

$$
\begin{gathered}
\sigma_{r}(r)=E_{\theta}\left[\frac{D_{1}}{k-\nu_{\theta r}} r^{k-1}+\frac{D_{2}}{k+\nu_{\theta r}} r^{-k-1}+\frac{\varepsilon_{r}^{t h}-\varepsilon_{\theta}^{t h}}{1-k^{2}}\right] \\
\sigma_{\theta}(r)=k E_{\theta}\left[\frac{D_{1}}{k-\nu_{\theta r}} r^{k-1}-\frac{D_{2}}{k+\nu_{\theta r}} r^{-k-1}+\frac{\varepsilon_{r}^{t h}-\varepsilon_{\theta}^{t h}}{k\left(1-k^{2}\right)}\right],
\end{gathered}
$$

For a free thermal contraction, the constants $D_{1}$ and $D_{2}$ are found by enforcing that the radial stress vanish at the inner and outer dimensions of the coil. For a detailed analysis of orthotropic coils in solenoids, the reader is referred to work by Markiewicz [6] and Gray [7].

Figure 4 shows the radial displacement of the coil base due to cooling from $295 \mathrm{~K}$ to $4.2 \mathrm{~K}$ with zero applied external loads. For this example the dimensions of the plane stress coil are chosen to be those at the top of the vector magnet coil where the inner radius is $22 \mathrm{~mm}$ and the outer radius is 71.5 $\mathrm{mm}$. For an isotropic material, if the radial displacement curve is extrapolated to zero radial distance, the radial displacement should vanish. For the orthotropic material considered here, this is not the case. The dashed line in figure 4 represents the effective slope of the radial displacement curve for the inner surface of the coil, which is considerably smaller than the slope of the displacement curve inside of the coil. In the inner surface the thermal contraction corresponds to that of an isotropic material with a thermal contraction of $0.15 \%$ between $295 \mathrm{~K}$ and $4.2 \mathrm{~K}$. On the outer surface the thermal contraction corresponds to that of an isotropic material with a thermal contraction of $0.31 \%$ between $295 \mathrm{~K}$ and $4.2 \mathrm{~K}$. This result is important for the design of magnet since it is desirable to have the coil remain in contact with the surrounding structure after the magnet is cold. The thermal deformation of the orthotropic coil is clearly different that that of a coil with assumed isotropic properties (where either the radial or azimuthal thermal strain is assumed). Figure 5 shows the stress in the radial and azimuthal directions due to the cool down of the coil under the plane stress assumption. The radial stress is tensile throughout the coil with a peak value of $19 \mathrm{MPa}$ while the azimuthal stress has a maximum tensile value (95 MPa) at the inner radius and a maximum compressive value (-46 MPa) at the outer radius.

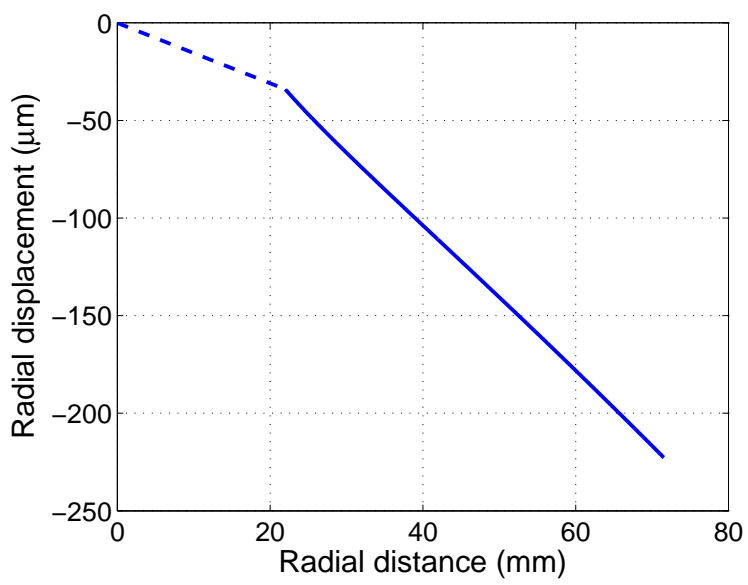

Fig. 4. Radial displacement of the coil base due to for cool down with zero applied external loads. The dashed line represents the effective slope of the radial displacement curve for the inner surface of the coil.

For comparison, figure 6 shows the FEM solution for the radial and azimuthal stress in the coil where the plane stress and cylindrical shape assumptions are removed. The FEM and analytical plane stress calculations are seen to match closely near the top of the coil where the inner and outer radius match 


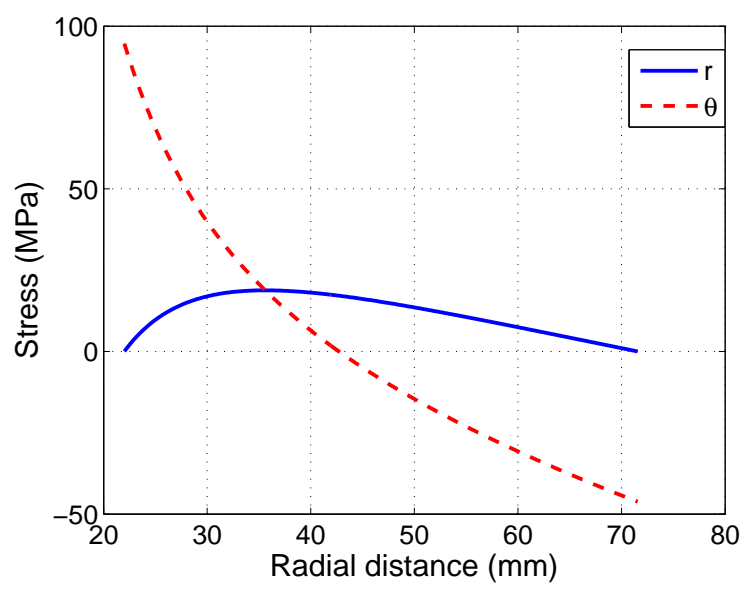

Fig. 5. Radial and azimuthal stress due to the cooling of the orthotropic coil under the plane stress assumption.

those used in the analytical result. As the coil becomes thinner towards the bottom the effect of the residual stress due to the orthotropic nature of the coil diminishes. With respect to the design and structural analysis of the magnet, this effect will lead to a shape change between the coil and the pocket in the monolithic structure during cool down.

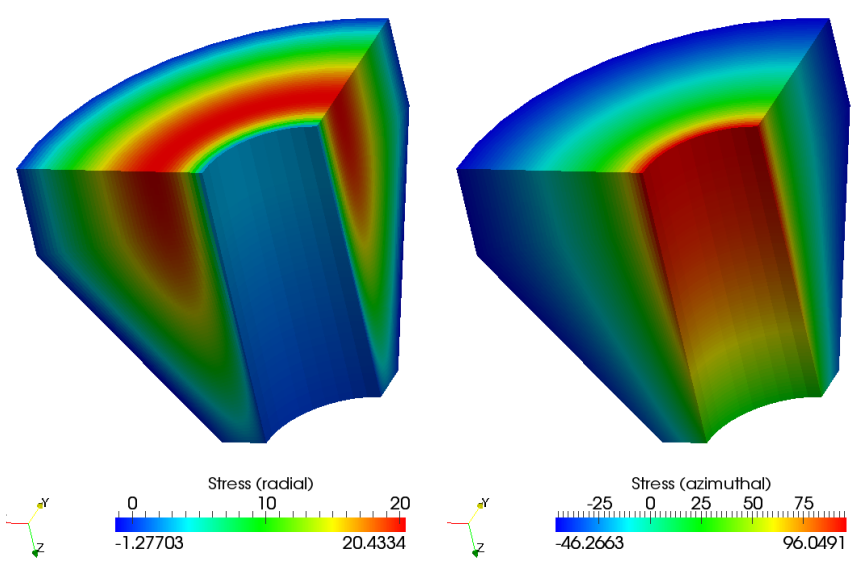

Fig. 6. Radial (left) and azimuthal (right) components of stress in the coil due to cool down from $295 \mathrm{~K}$ to $4.2 \mathrm{~K}$ with zero tractions on all boundaries.

\section{Structural Magnet Model}

For the structural analysis of the vector magnet the following components are considered: the coil, the pole, the yoke, as well as the internal monolithic structure. In the finite element model, all material interfaces are meshed with contact elements. The contact pairs that are modeled are: coilsupport structure, coil-pole, coil-yoke, pole-yoke, pole-support structure, and yoke-support structure. The behavior of the elements can be specified as either a stick-slip interface, a nonseparating interface (i.e. only motion in the tangential direction is allowed), or a fully glued interface. For the case where the magnetic field is oriented in one of the principal directions, a one-eighth model can be used due to symmetry considerations. A zero normal displacement boundary condition on the three flat lateral surfaces (x, y, and z planes) of the one-eighth model is used. The magnetic forces are calculated using TOSCA and applied to the structural ANSYS model on the pole and coil elements. A homogeneous temperature load is applied on every element in the model to simulate the cool down from room temperature to $4.2 \mathrm{~K}$. Figure 7 shows the results obtained for the contact pressure on the coil due to both the cool down and energization of the magnet. For this case, the entire base of the coil is in compression from the differential thermal strain between the Aluminum support structure and the coil. Only certain regions of the conical face of the coil remain in contact with the support structure after cool down and energization. The region of highest pressure on the coil is normal to the direction in which the field is oriented. This occurs since the coils are pulled towards each other in this direction.

ANSYS 11.0SP1

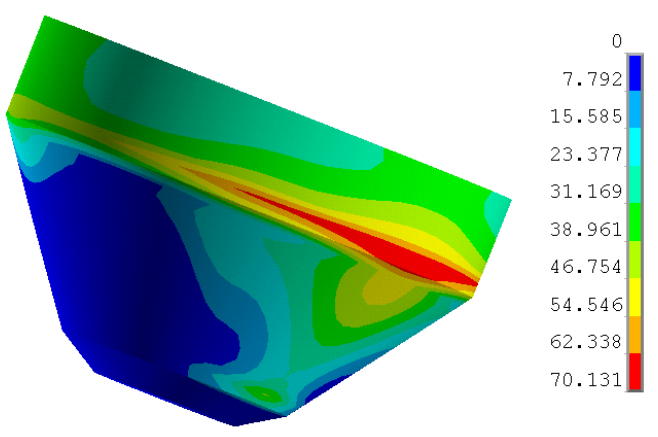

Fig. 7. Finite element solution for the contact pressure between the coil and the monolithic support structure after cool down and energization of the magnet. The units for pressure in the figure are in $\mathrm{MPa}$.

\section{CONCLUSION}

In this paper, the structural analysis for an eight pole superconducting magnet was presented. Calculations were performed to determine the orthotropic material properties of the coil. Through the plane stress analysis it was shown that the thermal deformation of the inner and outer radii of the coil does not correspond to that seen in an isotropic material where the thermal strain state is equivalent in both locations. Therefore, the inclusion of the orthotropic behavior is a critical component in order to determine the contact behavior of the coil-structure and coil-pole interfaces. The ANSYS model of the magnet allows for different contact scenarios at these interfaces. The optimal choice for having certain interfaces glued or allowed to slip is currently being investigated. The accuracy of the model is clearly dependent on the material properties of all of the components of the magnet. This includes the constituent materials in the coil. Since there is a fair amount of uncertainty in these properties, accurate measurements over the temperature range of interest are necessary.

\section{REFERENCES}

[1] E. Arenholz and S.O. Prestemon, "Design and performance of an eightpole resistive magnet for soft x-ray magnetic dichroism measurements", Review of Scientific Instruments, vol. 76, 083908, 2005. 
[2] D. Arbelaez et al., "Cable deformation simulation and a hierarchical framework for Nb3Sn rutherford cables", J. Phys.: Conf. Ser., vol. 234, $022002,2010$.

[3] N. Mitchell, "Finite element simulations of elasto-plastic processes in Nb3Sn strands", Cryogenics, vol. 45, no. 7, pp. 501-515, 2005.

[4] CTD-101 epoxy resin system data sheet, Composite Technology Development Inc., Lafayette, CO, 2002.

[5] C.L. Goodzeit, Superconducting accelerator magnets USPAS lecture notes, USPAS January 2001.

[6] w.D. Markiewicz et al., "Generalized plane strain analysis of superconducting solenoids", Journal of Applied Physics, vol. 86, no. 12, pp. 7039 - 7051, 1999.

[7] W.H. Gray and J.K. Ballou, "Electromechanical stress analysis of transversely isotropic solenoids", Journal of Applied Physics, vol. 48, no. 7, pp. 3100 - 3109, 1977.

Supported by the U.S. Department of Energy under Contract No.

DE-AC02-05CH11231. 


\section{LEGAL DISCLAIMER}

This document was prepared as an account of work sponsored by the United States Government. While this document is believed to contain correct information, neither the United States Government nor any agency thereof, nor The Regents of the University of California, nor any of their employees, makes any warranty, express or implied, or assumes any legal responsibility for the accuracy, completeness, or usefulness of any information, apparatus, product, or process disclosed, or represents that its use would not infringe privately owned rights. Reference herein to any specific commercial product, process, or service by its trade name, trademark, manufacturer, or otherwise, does not necessarily constitute or imply its endorsement, recommendation, or favoring by the United States Government or any agency thereof, or The Regents of the University of California. The views and opinions of authors expressed herein do not necessarily state or reflect those of the United States Government or any agency thereof or The Regents of the University of California. 